\title{
Imaging the Rapid Solidification of Metallic Alloys in the TEM
}

John D. Roehling ${ }^{1}$, Aurélien Perron ${ }^{1}$, Jean-Luc Fattebert ${ }^{2}$, Daniel R. Coughlin ${ }^{3}$, Paul J. Gibbs $^{3}$, John W. Gibbs ${ }^{3}$, Seth D. Imhoff ${ }^{3}$, Damien Tourret ${ }^{3}$, J. Kevin Baldwin ${ }^{4}$, Amy J. Clarke $^{3}$, Patrice E.A. Turchi ${ }^{1}$, and Joseph T. McKeown ${ }^{1}$

${ }^{1}$ Materials Science Division, Lawrence Livermore National Laboratory, Livermore, CA, USA

${ }^{2}$ Center for Applied Scientific Computing, Lawrence Livermore National Laboratory, Livermore, CA, USA

${ }^{3}$ Materials Science and Technology Division, Los Alamos National Laboratory, Los Alamos, NM, USA

${ }^{4}$ Center for Integrated Nanotechnologies, Los Alamos National Laboratory, Los Alamos, NM, USA

The macroscopic properties of a metal solidified from a liquid melt are strongly dependent on the final microstructure, which in turn is the result of the solidification conditions. With the growing popularity of laser-based additive manufacturing (AM), there is an increasing need to understand the microstructures that result from rapid solidification processes. Rapidly solidified alloy microstructures are typically far from equilibrium and therefore traditional thermodynamic approaches used to predict structure and composition (i.e., phase diagrams) must be extended to describe these deviations from equilibrium and ensuing metastable states. This work highlights progress toward corroborating predictive (phase-field) modeling capabilities [1] with in situ experimental observations [2] in order to better understand the non-equilibrium structures produced during rapid solidification following laser melting.

Identification of solidification modes and associated velocities using ex situ analyses after laser melting and rapid solidification usually represent averages for the complete liquidsolid transformation and fail to detect changes in velocity that are expected when transitions in the solidification mode occur. Models predicting the solidification mode can potentially bridge the gap, but direct experimental measurements of the kinetics and growth modes are needed to verify the modeling results. To directly observe rapid alloy solidification under laser-induced conditions that apply to AM processing, the dynamic transmission electron microscope (DTEM) at Lawrence Livermore National Lab (LLNL) was used in Movie Mode [3] (Figure 1). The Movie Mode capability at LLNL allows for single solidification events to be monitored in situ. The quasi-instantaneous velocity can be measured (Figure 2) over a given time domain, which would be inaccessible with lower temporal resolution techniques such as conventional TEM, and related directly to the observed solid-liquid interface evolution in the time-resolved images. Additionally, the ability to alter the power of the sample drive laser allows for a range of solidification 
velocities to be accessed. The resulting solidification structures are analyzed with grain orientation mapping and elemental analysis (EELS and EDS). Finally, the data are compared against phase-field modeling of solidification under different conditions.

\section{References}

[1] J.-L. Fattebert, M.E. Wickett, P.E.A. Turchi, Acta Materialia 62 (2014) 89.

[2] J.T. McKeown et. al, Acta Materialia 65 (2014) 56.

[3] T. LaGrange, B.W. Reed, D.J. Masiel, MRS Bulletin 40 (2015) 22.

[4] This work was performed under the auspices of the U.S. Department of Energy (DOE) by Lawrence Livermore National Laboratory under Contract DE-AC5207NA27344 and Los Alamos National Laboratory (LANL), operated by Los Alamos National Security, LLC, under Contract DE-AC52-06NA25396. Work at LLNL was funded by the Laboratory Directed Research and Development Program at LLNL under project tracking code 15-ERD-006. AJC, DRC, PJG, JWG, SDI, and DT were supported by AJC's Early Career award from the U.S. DOE, Office of Basic Energy Sciences, Division of Materials Sciences and Engineering. Work at LANL was performed, in part, at the Center for Integrated Nanotechnologies, an Office of Science User Facility operated for the U.S. DOE Office of Science.

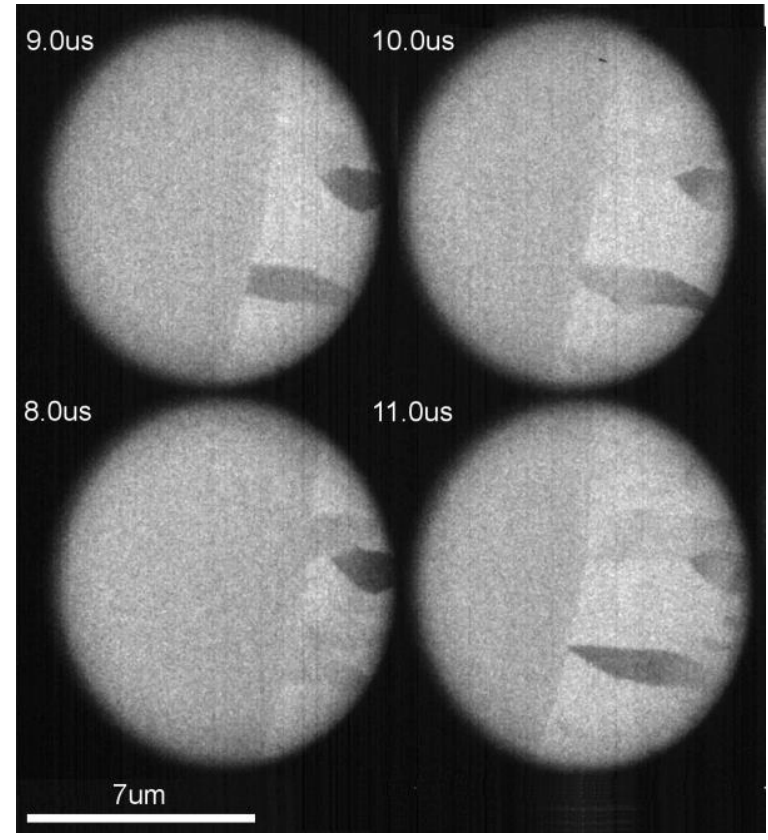

Figure 1: Dynamic TEM Movie Mode images of an Al-Si film on amorphous $\mathrm{SiN}_{\mathrm{x}}$, microseconds after induced laser melting at the edge of the solidifying melt pool.

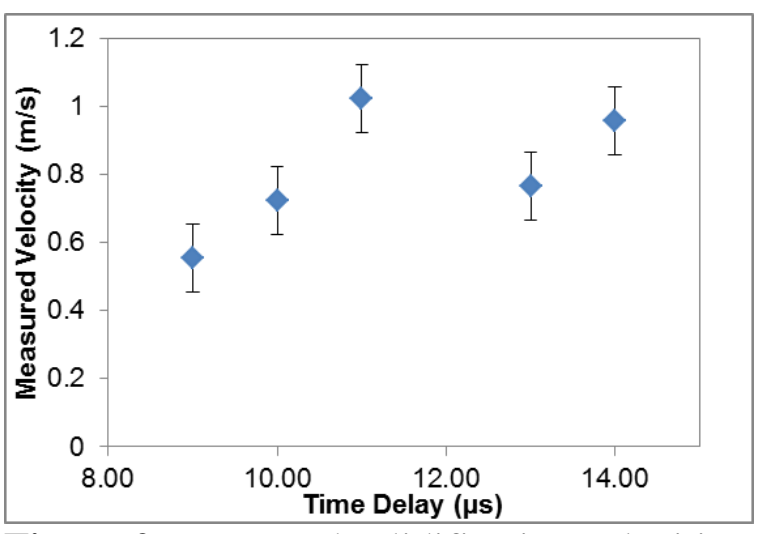

Figure 2: Measured solidification velocities from the DTEM Movie Mode acquisition shown in Figure 1. 